\title{
Axisymmetric motion of a second order viscous fluid in a circular straight tube under pressure gradients varying exponentially with time
}

\author{
F. Carapau ${ }^{1} \&$ A. Sequeira ${ }^{2}$ \\ ${ }^{1}$ Department of Mathematics and CIMA/UE, \\ University of Évora, Portugal \\ ${ }^{2}$ Department of Mathematics and CEMAT/IST, IST, Portugal
}

\begin{abstract}
The aim of this paper is to analyze the axisymmetric unsteady flow of a nonNewtonian incompressible second order fluid in a straight rigid and impermeable tube with circular cross-section of constant radius. To study this problem, we use the one dimensional (1D) nine-directors Cosserat theory approach which reduces the exact three-dimensional equations to a system depending only on time and on a single spatial variable. From this system we obtain the relationship between mean pressure gradient and volume flow rate over a finite section of the tube. Assuming that the pressure gradient rises and falls exponentially with time, the 3D exact solution for unsteady volume flow rate is compared with the corresponding 1D solution obtained by the Cosserat theory using nine directors.

Keywords: Cosserat theory, nine directors, unsteady rectilinear flow, axisymmetric motion, pressure gradient, second order fluid.
\end{abstract}

\section{Introduction}

A possible simplification to a three-dimensional model for an incompressible viscous fluid inside a domain is to consider the evolution of average flow quantities using simpler one-dimensional models. Usually, in the case of flow in a tube, the classical 1D models are obtained by imposing additional assumptions and integrating both the equations of conservation of linear momentum and mass over the cross section of the tube. Here, we introduce a 1D model for non-Newtonian Rivlin-Ericksen fluids of second order in an axisymmetric tube, based on the nine- 
director approach developed by Caulk and Naghdi [4]. This theory includes an additional structure of directors (deformable vectors) assigned to each point on a space curve (Cosserat curve), where a three-dimensional system of equations is replaced by a one-dimensional system depending on time and on a single spatial variable. The use of directors in continuum mechanics goes back to Duhen [7] who regards a body as a collection of points together with associated directions. Theories based on such a model of an oriented medium were further developed by Cosserat and Cosserat [6] and have also been used by several authors in studies of rods, plates and shells (see e.g. Ericksen and Truesdell [8], Truesdell and Toupin [17], Green and Naghdi [10,11] and Naghdi [13]). An analogous hierarchial theory for unsteady and steady flows has been developed by Caulk and Naghdi [4] in straight pipes of circular cross-section and by Green and Naghdi [12] in channels. The same theory was applied to unsteady viscous fluid flow in curved pipes of circular and elliptic cross-section by Green et al. [9]. Recently, the ninedirector theory has been applied to blood flow in the arterial system by Robertson and Sequeira [16] and also by Carapau and Sequeira [2, 3], considering Newtonian and shear-thinning flows, respectively. The relevance of using a theory of directed curves is not in regarding it as an approximation to 3D equations, but rather in their use as independent theories to predict some of the main properties of the three-dimensional problems. Advantages of the director theory include: (i) the theory incorporates all components of the linear momentum; (ii) it is a hierarchical theory, making it possible to increase the accuracy of the model; (iii) there is no need for closure approximations; (iv) invariance under superposed rigid body motions is satisfied at each order and (v) the wall shear stress enters directly in the formulation as a dependent variable.

This paper deals with the study of the initial boundary value problem for an incompressible homogeneous second order fluid model in a straight circular rigid and impermeable tube with constant radius, where the fluid velocity field, given by the director theory, can be approximated by the following finite series:

$$
\boldsymbol{v}^{*}=\boldsymbol{v}+\sum_{N=1}^{k} x_{\alpha_{1}} \ldots x_{\alpha_{N}} \boldsymbol{W}_{\alpha_{1} \ldots \alpha_{N}}
$$

with

$$
\boldsymbol{v}=v_{i}(z, t) \boldsymbol{e}_{i}, \quad \boldsymbol{W}_{\alpha_{1} \ldots \alpha_{N}}=W_{\alpha_{1} \ldots \alpha_{N}}^{i}(z, t) \boldsymbol{e}_{i},
$$

(latin indices subscript take the values $1,2,3$; greek indices subscript 1,2 , and the usual summation convention is employed over a repeated index). Here, $v$ represents the velocity along the axis of symmetry $z$ at time $t, x_{\alpha_{1}} \ldots x_{\alpha_{N}}$ are the polynomial weighting functions with order $k$ (this number identifies the order of hierarchical theory and is related to the number of directors), the vectors $\boldsymbol{W}_{\alpha_{1} \ldots \alpha_{N}}$ are the director velocities which are symmetric with respect to their indices and $\boldsymbol{e}_{i}$ are the associated unit basis vectors. When we use the director theory, the 3D system of equations governing the fluid motion is replaced by a system which depends only on a single spatial and time variables, as previously mentioned. From this new 
system, we obtain the unsteady relationship between mean pressure gradient and volume flow rate, and the correspondent equation for the wall shear stress.

The aim of this paper is to develop a nine-director theory $(k=3$ in equation $(1))$ for the unsteady flow of a second order fluid in a straight tube with constant radius, to compare the corresponding volume flow rate with the 3D exact solution given by Soundalgekar [15], when the pressure gradient rises and falls exponentially with time.

\section{Equations of motion}

We consider a homogeneous fluid moving within a circular straight and impermeable tube, the domain $\Omega$ (see fig. 1 ) contained in $\mathbb{R}^{3}$. Its boundary $\partial \Omega$ is composed by, the proximal cross-section $\Gamma_{1}$, the distal cross-section $\Gamma_{2}$ and the lateral wall of the tube, denoted by $\Gamma_{w}$.

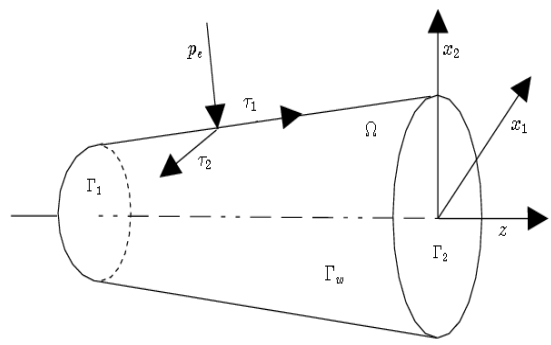

Figure 1: Fluid domain $\Omega$ with the components of the surface traction vector $\tau_{1}, \tau_{2}$ and $p_{e}$.

Let $x_{i}(i=1,2,3)$ be the rectangular Cartesian coordinates and for convenience set $x_{3}=z$. Consider the axisymmetric motion of an incompressible fluid without body forces, inside a straight circular tube, about the $z$ axis and let $\phi(z, t)$ denote the radius of that surface at $z$ and time $t$. Using the notation adopted in Naghdi $e t$ al. $[4,9]$, the three-dimensional equations governing the fluid motion are given by

$$
\begin{cases}\rho\left(\frac{\partial \boldsymbol{v}^{*}}{\partial t}+v_{, i}^{*} \boldsymbol{v}_{i}^{*}\right)=t_{i, i}, & \\ v_{i, i}^{*}=0, & \text { in } \Omega \times(0, T), \\ t_{i}=-p^{*} \boldsymbol{e}_{i}+\sigma_{i j} \boldsymbol{e}_{j}, \boldsymbol{t}=\vartheta_{i}^{*} t_{i}, & \end{cases}
$$

with the initial condition

$$
\boldsymbol{v}^{*}(x, 0)=\boldsymbol{v}_{0}(x) \text { in } \Omega,
$$


and the boundary condition

$$
\boldsymbol{v}^{*}(x, t)=0 \text { on } \Gamma_{w} \times(0, T),
$$

where $\boldsymbol{v}^{*}=v_{i}^{*} \boldsymbol{e}_{i}$ is the velocity field and $\rho$ is the constant fluid density. Equation $(3)_{1}$ represents the balance of linear momentum and $(3)_{2}$ is the incompressibility condition. In equation $(3)_{3}, p^{*}$ is the pressure, $\sigma_{i j}$ are the components of the extra stress tensor, $\boldsymbol{t}$ denotes the stress vector on the surface whose outward unit normal is $\vartheta^{*}=\vartheta_{i}^{*} \boldsymbol{e}_{i}$, and $t_{i}$ are the components of $\boldsymbol{t}$.

For a general incompressible Rivlin-Ericksen fluid of second order, the components of the extra stress tensor, in the constitutive equation $(3)_{3}$, are given by (see e.g. Coleman and Noll [5])

$$
\sigma_{i j}=\mu A_{i j}+\alpha_{1} S_{i j}+\alpha_{2} A_{i k} A_{k j}, \quad i, j, k=1,2,3
$$

where $\mu$ is the constant viscosity, $\alpha_{1}, \alpha_{2}$ are material constants (normal stress mod$u l i)$ and $A_{i j}, S_{i j}$ are the first two Rivlin-Ericksen tensors, defined by (see Rivlin and Ericksen [14])

$$
A_{i j}=\frac{\partial v_{i}^{*}}{\partial x_{j}}+\frac{\partial v_{j}^{*}}{\partial x_{i}}
$$

and

$$
S_{i j}=\frac{\partial A_{i j}}{\partial t}+v_{k}^{*} \frac{\partial A_{i j}}{\partial x_{k}}+A_{i k} \frac{\partial v_{k}^{*}}{\partial x_{j}}+\frac{\partial v_{k}^{*}}{\partial x_{i}} A_{k j} .
$$

Note that, if $\alpha_{1}=\alpha_{2}=0$ in equation (6) we obtain the classical Newtonian incompressible model.

We assume that the lateral surface $\Gamma_{w}$ of the axisymmetric tube is defined by

$$
\phi^{2}=x_{\alpha} x_{\alpha},
$$

and the components of the outward unit normal to this surface are

$$
\vartheta_{\alpha}^{*}=\frac{x_{\alpha}}{\phi\left(1+\phi_{z}^{2}\right)^{1 / 2}}, \quad \vartheta_{3}^{*}=-\frac{\phi_{z}}{\left(1+\phi_{z}^{2}\right)^{1 / 2}}
$$

where the subscript variable denotes partial differentiation. Since equation (9) defines a material surface, the velocity field must satisfy the kinematic condition

$$
\phi \phi_{t}+\phi \phi_{z} v_{3}^{*}-x_{\alpha} v_{\alpha}^{*}=0
$$

on the boundary (9).

Averaged quantities such as flow rate and average pressure are needed to study 1D models, in particular the unsteady relationship between mean pressure gradient and volume flow rate over a finite section of the tube. Consider $S(z, t)$ as a generic axial section of the tube at time $t$ defined by the spatial variable $z$ and bounded by 
the circle defined in $(9)$ and let $A(z, t)$ be the area of this section $S(z, t)$. Then, the volume flow rate $Q$ is defined by

$$
Q(z, t)=\int_{S(z, t)} v_{3}^{*}\left(x_{1}, x_{2}, z, t\right) d a,
$$

and the average pressure $\bar{p}$, by

$$
\bar{p}(z, t)=\frac{1}{A(z, t)} \int_{S(z, t)} p^{*}\left(x_{1}, x_{2}, z, t\right) d a .
$$

In the sequel, this general framework will be applied to the specific case of the Cosserat nine-director theory in a rigid tube, i.e. $\phi=\phi(z)$. Using condition (1) it follows from Caulk and Naghdi [4] that the approximation for the threedimensional velocity field $\boldsymbol{v}^{*}$ is given by

$$
\begin{aligned}
\boldsymbol{v}^{*} & =\left[x_{1}\left(1-\frac{x_{1}^{2}+x_{2}^{2}}{\phi^{2}}\right) \frac{2 \phi_{z} Q}{\pi \phi^{3}}\right] \boldsymbol{e}_{1}+\left[x_{2}\left(1-\frac{x_{1}^{2}+x_{2}^{2}}{\phi^{2}}\right) \frac{2 \phi_{z} Q}{\pi \phi^{3}}\right] \boldsymbol{e}_{2} \\
& +\left[\frac{2 Q}{\pi \phi^{2}}\left(1-\frac{x_{1}^{2}+x_{2}^{2}}{\phi^{2}}\right)\right] \boldsymbol{e}_{3}
\end{aligned}
$$

where the volume flow rate $Q(t)$ is

$$
Q(t)=\frac{\pi}{2} \phi^{2}(z) v_{3}(z, t)
$$

We remark that the initial condition (4) is satisfied when $Q(0)=$ ct. Also, from Caulk and Naghdi [4] the stress vector on the lateral surface $\Gamma_{w}$ is given by

$$
\begin{aligned}
\boldsymbol{t}_{w} & =\left[\frac{1}{\phi\left(1+\phi_{z}^{2}\right)^{1 / 2}}\left(\tau_{1} x_{1} \phi_{z}-p_{e} x_{1}-\tau_{2} x_{2}\left(1+\phi_{z}^{2}\right)^{1 / 2}\right)\right] \boldsymbol{e}_{1} \\
& +\left[\frac{1}{\phi\left(1+\phi_{z}^{2}\right)^{1 / 2}}\left(\tau_{1} x_{2} \phi_{z}-p_{e} x_{2}+\tau_{2} x_{1}\left(1+\phi_{z}^{2}\right)^{1 / 2}\right)\right] \boldsymbol{e}_{2} \\
& +\left[\frac{1}{\left(1+\phi_{z}^{2}\right)^{1 / 2}}\left(\tau_{1}+p_{e} \phi_{z}\right)\right] \boldsymbol{e}_{3} .
\end{aligned}
$$

Instead of satisfying the momentum equation $(3)_{1}$ pointwise in the fluid, we impose the following integral conditions

$$
\begin{gathered}
\int_{S(z, t)}\left[t_{i, i}-\rho\left(\frac{\partial \boldsymbol{v}^{*}}{\partial t}+\boldsymbol{v}_{, i}^{*} v_{i}^{*}\right)\right] d a=0, \\
\int_{S(z, t)}\left[t_{i, i}-\rho\left(\frac{\partial \boldsymbol{v}^{*}}{\partial t}+\boldsymbol{v}_{, i}^{*} v_{i}^{*}\right)\right] x_{\alpha_{1}} \ldots x_{\alpha_{N}} d a=0,
\end{gathered}
$$

where $N=1,2,3$. 
Using the divergence theorem and integration by parts, equations $(17)-(18)$ for nine directors, can be reduced to the four vector equations:

$$
\frac{\partial \boldsymbol{n}}{\partial z}+\boldsymbol{f}=\boldsymbol{a}, \frac{\partial \boldsymbol{m}^{\alpha_{1} \ldots \alpha_{N}}}{\partial z}+\boldsymbol{l}^{\alpha_{1} \ldots \alpha_{N}}=\boldsymbol{k}^{\alpha_{1} \ldots \alpha_{N}}+\boldsymbol{b}^{\alpha_{1} \ldots \alpha_{N}}
$$

where $\boldsymbol{n}, \boldsymbol{k}^{\alpha_{1} \ldots \alpha_{N}}, \boldsymbol{m}^{\alpha_{1} \ldots \alpha_{N}}$ are resultant forces defined by

$$
\begin{gathered}
\boldsymbol{n}=\int_{S} \boldsymbol{t}_{3} d a, \quad \boldsymbol{k}^{\alpha}=\int_{S} \boldsymbol{t}_{\alpha} d a, \boldsymbol{k}^{\alpha \beta}=\int_{S}\left(\boldsymbol{t}_{\alpha} x_{\beta}+\boldsymbol{t}_{\beta} x_{\alpha}\right) d a \\
\boldsymbol{k}^{\alpha \beta \gamma}=\int_{S}\left(\boldsymbol{t}_{\alpha} x_{\beta} x_{\gamma}+\boldsymbol{t}_{\beta} x_{\alpha} x_{\gamma}+\boldsymbol{t}_{\gamma} x_{\alpha} x_{\beta}\right) d a \\
\boldsymbol{m}^{\alpha_{1} \ldots \alpha_{N}}=\int_{S} \boldsymbol{t}_{3} x_{\alpha_{1}} \ldots x_{\alpha_{N}} d a .
\end{gathered}
$$

The quantities $\boldsymbol{a}$ and $\boldsymbol{b}^{\alpha_{1} \ldots \alpha_{N}}$ are inertia terms written as follows

$$
\begin{gathered}
\boldsymbol{a}=\int_{S} \rho\left(\frac{\partial \boldsymbol{v}^{*}}{\partial t}+\boldsymbol{v}_{, i}^{*} v_{i}^{*}\right) d a \\
\boldsymbol{b}^{\alpha_{1} \ldots \alpha_{N}}=\int_{S} \rho\left(\frac{\partial \boldsymbol{v}^{*}}{\partial t}+\boldsymbol{v}_{, i}^{*} v_{i}^{*}\right) x_{\alpha_{1}} \ldots x_{\alpha_{N}} d a,
\end{gathered}
$$

and $\boldsymbol{f}, \boldsymbol{l}^{\alpha_{1} \ldots \alpha_{N}}$, which arise due to surface traction on the lateral boundary, are given by

$$
\boldsymbol{f}=\int_{\partial S}\left(1+\phi_{z}^{2}\right)^{1 / 2} \boldsymbol{t}_{w} d s, \boldsymbol{l}^{\alpha_{1} \ldots \alpha_{N}}=\int_{\partial S}\left(1+\phi_{z}^{2}\right)^{1 / 2} \boldsymbol{t}_{w} x_{\alpha_{1}} \ldots x_{\alpha_{N}} d s .
$$

The equation relating the mean pressure gradient with the volume flow rate will be obtained using these quantities.

\section{Results and discussion}

We consider the case of a straight circular rigid and impermeable walled tube with constant radius, i.e. $\phi=c t$. Replacing the results $(20)-(25)$ obtained for the nine-director model into equations (19), we get the unsteady relationship

$$
\bar{p}_{z}(z, t)=-\frac{8 \mu}{\pi \phi^{4}} Q(t)-\frac{4 \rho}{3 \pi \phi^{2}}\left(1+6 \frac{\alpha_{1}}{\rho \phi^{2}}\right) \dot{Q}(t),
$$

were the notation $\dot{Q}$ is used for time differentiation. Flow separation occurs when the axial component $\tau_{1}$ of the stress vector on the lateral surface (cf. (16)) is in 
the direction of the flow, i.e. $\tau_{1}>0$. The expression for the wall shear stress $\tau_{1}$ is given by

$$
\tau_{1}=\frac{4 \mu}{\pi \phi^{3}} Q(t)+\frac{\rho}{6 \pi \phi}\left(1+24 \frac{\alpha_{1}}{\rho \phi^{2}}\right) \dot{Q}(t) .
$$

Integrating equation (26), over a finite section of the tube, between $z_{1}$ and position $z_{2}\left(z_{1}<z_{2}\right)$, we get the mean pressure gradient

$$
G(t)=\frac{\bar{p}\left(z_{1}, t\right)-\bar{p}\left(z_{2}, t\right)}{z_{2}-z_{1}}=\frac{8 \mu}{\pi \phi^{4}} Q(t)+\frac{4 \rho}{3 \pi \phi^{2}}\left(1+6 \frac{\alpha_{1}}{\rho \phi^{2}}\right) \dot{Q}(t) .
$$

Now, let us consider the following dimensionless variables

$$
\hat{z}=\frac{z}{\phi}, \hat{t}=\omega_{0} t, \hat{Q}=\frac{2 \rho}{\pi \phi \mu} Q, \quad \hat{\bar{p}}=\frac{\phi^{2} \rho}{\mu^{2}} \bar{p},
$$

where $\phi$ is the characteristic radius of the tube and $\omega_{0}$ is the characteristic frequency for unsteady flow. Substituting the new variables (29) into equation (26), we obtain

$$
\hat{\bar{p}}_{\hat{z}}=-4 \hat{Q}(\hat{t})-\frac{2}{3}\left(1+6 \mathcal{W}_{e}\right) \mathcal{W}_{0}^{2} \dot{\hat{Q}}(\hat{t}),
$$

where $\mathcal{W}_{0}=\phi_{0} \sqrt{\rho \omega_{0} / \mu}$ is the Womersley number and $\mathcal{W}_{e}=\alpha_{1} /\left(\rho \phi^{2}\right)$ is a viscoelastic parameter, also called the Weissenberg number. The dimensionless number $\mathcal{W}_{0}$ is the most commonly used parameter to reflect the unsteady pulsatility of the flow. Integrating (30) over a finite section of the tube between $\hat{z}_{1}$ and $\hat{z}_{2}$, we get the relationship between mean pressure gradient and volume flow rate given by

$$
\hat{\bar{G}}(\hat{t})=4 \hat{Q}(\hat{t})+\frac{2}{3}\left(1+6 \mathcal{W}_{e}\right) \mathcal{W}_{0}^{2} \dot{\hat{Q}}(\hat{t}) .
$$

Moreover, the dimensionless form of equation (27) is

$$
\hat{\tau}_{1}=2 \hat{Q}(\hat{t})+\frac{1}{12}\left(1+24 \mathcal{W}_{e}\right) \mathcal{W}_{0}^{2} \dot{\hat{Q}}(\hat{t}) \quad \text { with } \quad \hat{\tau}_{1}=\frac{\phi^{2} \rho}{\mu^{2}} \tau_{1} .
$$

Next we compare the exact solution for a rectilinear motion (given by Soundalgekar [15]) with the solution obtained by the nine-director theory in a straight circular rigid tube with constant radius $\phi$, when the pressure gradient rises and falls exponentially with time.

\subsection{Pressure gradient rising exponentially with time}

Let us assume the following pressure gradient

$$
-\frac{1}{\rho} p_{z}^{*}=k \exp \left(\theta^{2} t\right)
$$

where $k$ and $\theta$ are constants, with $\theta^{2}$ being the characteristic frequency. Then the velocity field solution obtained by Soundalgekar [15], with $|\beta \phi| \ll 1$ and 
$\left|\beta \sqrt{x_{1}^{2}+x_{2}^{2}}\right| \ll 1$, is given by

$$
v_{3}^{*}=\left[\frac{k \beta^{2}}{4 \theta^{2}}\left(\phi^{2}-\left(x_{1}^{2}+x_{2}^{2}\right)\right) \exp \left(\theta^{2} t\right)\right] \boldsymbol{e}_{3},
$$

where $\beta^{2}=\rho \theta^{2} /\left(\mu+\alpha_{1} \theta^{2}\right)$. From (33) the volume flow rate reduces to

$$
Q(t)=\frac{k \phi^{4} \pi \rho}{8\left(\mu+\alpha_{1} \theta^{2}\right)} \exp \left(\theta^{2} t\right) .
$$

Using the nondimensional variables

$$
\hat{t}=\theta^{2} t, \quad \hat{Q}=\frac{8 \mu}{k \phi^{4} \pi \rho} Q,
$$

into equation (34), we obtain the nondimensional volume flow rate

$$
\hat{Q}(\hat{t})=\frac{1}{1+\mathcal{W}_{e} \mathcal{W}_{0}^{2}} \exp (\hat{t}) .
$$

In view of the pressure gradient (32) and equation (31) given by the nine-director theory, we get the following nondimensional volume flow rate

$$
\begin{aligned}
\hat{Q}(\hat{t}) & =\frac{1}{12+2 \mathcal{W}_{0}^{2}+12 \mathcal{W}_{0}^{2} \mathcal{W}_{e}}[3 \exp (\hat{t}) \\
& \left.+\exp \left(\frac{-6 \hat{t}}{\mathcal{W}_{0}^{2}+6 \mathcal{W}_{0}^{2} \mathcal{W}_{e}}\right)\left(\frac{9+2 \mathcal{W}_{0}^{2}+9 \mathcal{W}_{0}^{2} \mathcal{W}_{e}}{1+\mathcal{W}_{e} \mathcal{W}_{0}^{2}}\right)\right] .
\end{aligned}
$$

Next, we compare the relationship between the volume flow rates (36) and (37), for a fixed Womersley number and different values of the Weissenberg number. Results in fig. 2 show that for $\mathcal{W}_{0}=0.5$, the solutions $(36)$ and (37) have the same qualitative behavior for increasing Weissenberg numbers, but show a large deviation in time. Numerical simulations for different Womersley numbers have shown similar results.

\subsection{Pressure gradient falling exponentially with time}

We consider the pressure gradient given by

$$
-\frac{1}{\rho} p_{z}^{*}=k \exp \left(-\theta^{2} t\right)
$$

The velocity field solution obtained by Soundalgekar [15], with $|\zeta \phi| \ll 1$ and $\left|\zeta \sqrt{x_{1}^{2}+x_{2}^{2}}\right| \ll 1$, is given by

$$
v_{3}^{*}=\left[\frac{k \zeta^{2}}{4 \theta^{2}}\left(\phi^{2}-\left(x_{1}^{2}+x_{2}^{2}\right)\right) \exp \left(-\theta^{2} t\right)\right] \boldsymbol{e}_{3},
$$



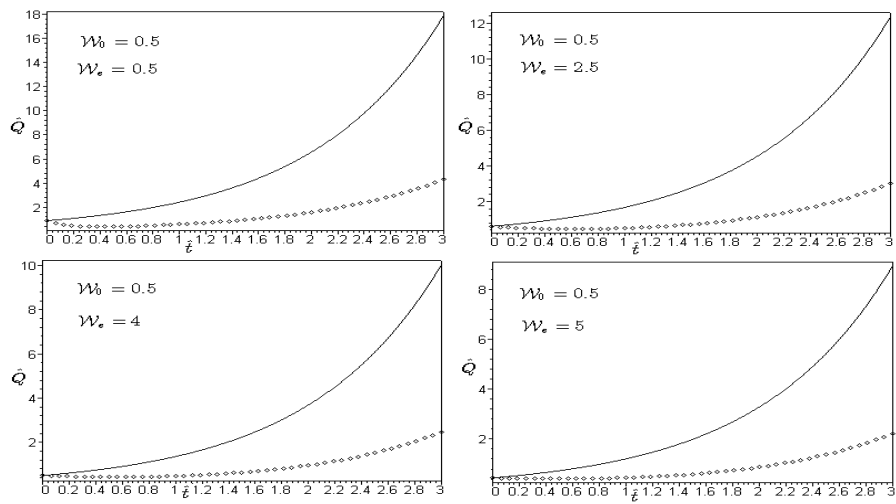

Figure 2: Volume flow rate when the pressure gradient is rising exponentially with time for different values of Weissenberg number, with fixed Womersley number $\left(\mathcal{W}_{0}=0.5\right)$ : nine-directors solution $(37)$ (dotted line) and exact 3D solution (36) (dark line).

where $\zeta^{2}=\rho \theta^{2} /\left(\mu-\alpha_{1} \theta^{2}\right)$ and the corresponding volume flow rate is

$$
Q(t)=\frac{k \phi^{4} \pi \rho}{8\left(\mu-\alpha_{1} \theta^{2}\right)} \exp \left(-\theta^{2} t\right) .
$$

Using the dimensionless variables (35) into the preceding equation, we obtain

$$
\hat{Q}(\hat{t})=\frac{1}{1-\mathcal{W}_{e} \mathcal{W}_{0}^{2}} \exp (-\hat{t}) .
$$

Taking into account the pressure gradient (38) and the nine-directors equation (31), we obtain the following nondimensional volume flow rate

$$
\begin{aligned}
\hat{Q}(\hat{t}) & =\frac{1}{-12+2 \mathcal{W}_{0}^{2}+12 \mathcal{W}_{0}^{2} \mathcal{W}_{e}}[-3 \exp (-\hat{t}) \\
& \left.+\exp \left(\frac{-6 \hat{t}}{\mathcal{W}_{0}^{2}+6 \mathcal{W}_{0}^{2} \mathcal{W}_{e}}\right)\left(\frac{9-2 \mathcal{W}_{0}^{2}-9 \mathcal{W}_{0}^{2} \mathcal{W}_{e}}{\mathcal{W}_{e} \mathcal{W}_{0}^{2}-1}\right)\right] .
\end{aligned}
$$

In fig. 3 we illustrate the behavior of the nine-directors solution versus the exact $3 \mathrm{D}$ solution, for a fixed Womersley number and different values of the Weissenberg number. The solutions show a small deviation for short times and approach asymptotically when time increases. Several numerical tests have also been performed for other Womersley numbers showing similar results.

\section{Conclusion}

The Cosserat nine-director theory applied to the axisymmetric unsteady flow behavior of a second order fluid in a straight tube, with uniform circular crosssection, has been evaluated by comparing its solution with the 3D exact solution 

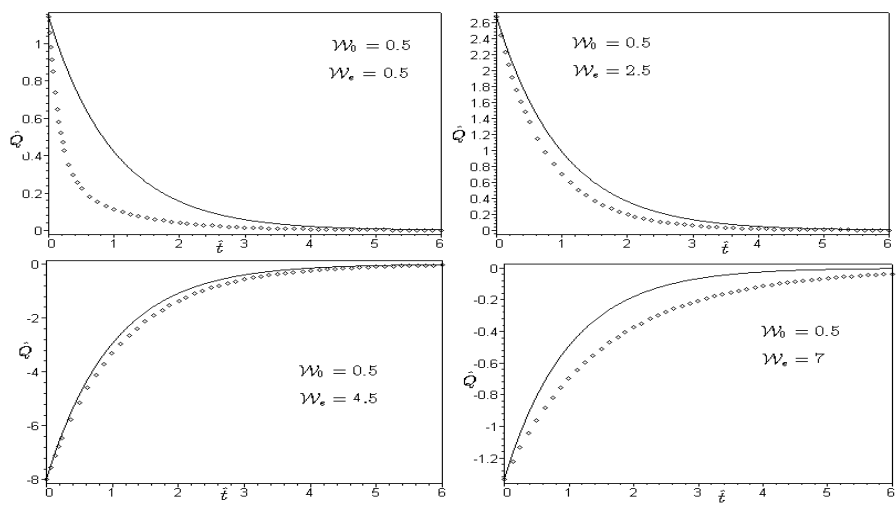

Figure 3: Volume flow rate with pressure gradient is falling exponentially with time for different values of Weissenberg number, with fixed Womersley number $\left(\mathcal{W}_{0}=0.5\right)$ : nine-directors solution $(42)$ (dotted line) and exact 3D solution (41) (dark line).

for unsteady flows, given by Soundalgekar [15]. For fixed Womersley number, when the pressure gradient rises exponentially with time, both solutions have the same qualitative behavior, but show a large deviation for increasing time. However, when the pressure gradient is falling exponentially with time, the solutions show a small deviation for short times and approach asymptotically in time. One of the important extensions of this work is the application of the Cosserat $1 \mathrm{D}$ theory to non-Newtonian second order fluids in a straight tube with non-constant radius. A more detailed discussion of this issue can be found in [1].

\section{Acknowledgements}

The authors are grateful to Professor A. M. Robertson (Univ. Pittsburgh, USA) for helpful discussions. This work has been partially supported by projects POCTI/MAT/41898/2001, HPRN-CT-2002-00270 of the European Union and by the research centers CEMAT-IST and CIMA-UE, through FCT's funding program.

\section{References}

[1] Carapau, F., Development of 1D Fluid Models Using the Cosserat Theory. Numerical Simulations and Applications to Haemodynamics, $\mathrm{PhD}$ Thesis, IST, Lisbon, Portugal, 2005.

[2] Carapau, F., \& Sequeira, A., Axisymmetric flow of a generalized Newtonian fluid in a straight pipe using a director theory approach, Proceedings of the 8th WSEAS International Conference on Applied Mathematics, pp. 303-308, 2005. 
[3] Carapau, F., \& Sequeira, A., 1D Models for blood flow in small vessels using the Cosserat theory, WSEAS Transactions on Mathematics, Issue 1, Vol.5, pp. 54-62, 2006.

[4] Caulk, D.A., \& Naghdi, P.M., Axisymmetric motion of a viscous fluid inside a slender surface of revolution, Journal of Applied Mechanics, Vol.54, pp. 190-196, 1987.

[5] Coleman, B.D., \& Noll, W., An approximation theorem for functionals with applications in continuum mechanics, Arch. Rational Mech. Anal., Vol.6, pp. 355-370, 1960.

[6] Cosserat, E. \& Cosserat, F., Sur la théorie des corps minces, Compt. Rend., Vol.146, pp. 169-172, 1908.

[7] Duhem, P., Le potentiel thermodynamique et la pression hydrostatique, Ann. 'Ecole Norm, Vol.10, pp. 187-230, 1893.

[8] Ericksen, J.L. \& Truesdell, C., Exact theory of stress and strain in rods and shells, Arch. Rational Mech. Anal., Vol.1, pp. 295-323, 1958.

[9] Green, A.E. \& Naghdi, P.M., A direct theory of viscous fluid flow in pipes: II Flow of incompressible viscous fluid in curved pipes, Phil. Trans. R. Soc. Lond. A, Vol.342, pp. 543-572, 1993.

[10] Green, A.E., Laws, N. \& Naghdi, P.M., Rods, plates and shells, Proc. Camb. Phil. Soc., Vol.64, pp. 895-913, 1968.

[11] Green, A.E., Naghdi, P.M. \& Wenner, M.L., On the theory of rods II. Developments by direct approach, Proc. R. Soc. Lond. A, Vol.337, pp. 485-507, 1974.

[12] Green, A.E. \& Naghdi, P.M., A direct theory of viscous fluid flow in channels, Arch. Ration. Mech. Analysis, Vol.86, pp. 39-63, 1984.

[13] Naghdi, P.M., The Theory of Shells and Plates, Flügg's Handbuch der Physik, Vol. VIa/2, Berlin, Heidelberg, New York: Springer-Verlag, pp. 425-640, 1972.

[14] Rivlin, R.S., \& Ericksen, J.L., Stress-deformation relations for isotropic materials, J. Rational Mech. Anal., Vol.4, pp. 323-425, 1955.

[15] Soundalgekar, V.M., The flow of a second order viscous fluid in a circular tube under pressure gradients varying exponentially with time, Indian J. Phys, Vol.46, pp. 250-254, 1972.

[16] Robertson, A.M. \& Sequeira, A., A director theory approach for modeling blood flow in the arterial system: An alternative to classical 1D models, Mathematical Models \& Methods in Applied Sciences, Vol.15, nr.6, pp. 871906, 2005.

[17] Truesdell, C. \& Toupin, R., The Classical Field Theories of Mechanics, Handbuch der Physik, Vol. III, pp. 226-793, 1960. 